\title{
Research and Development of Grid Model Online in E Imported to DIgSILENT
}

\author{
Jiutong LIN ${ }^{1, a}$, Dongying ZHANG ${ }^{1, b}$, Jianyang HUANG $^{1, c}$, Yuehui HUANG ${ }^{2, d}$, \\ Dewei LIU ${ }^{2, \text { e }}$ \\ ${ }^{1}$ North China Electric Power University, Beijing, 102206, China \\ ${ }^{2}$ China Electric Power Research Institute, Beijing, 100085, China \\ aemail: jtlin1020@hotmail.com, bemail: zdyingmail@163.com, ${ }^{\mathrm{C} e m a i l: ~ j i a n y a n g h @ 163 . c o m, ~ d e m a i l: ~}$ \\ huangyh@epri.sgcc.com.cn, email: liudw@epri.sgcc.com.cn
}

Keywords: Power System Simulation; E text; DIgSILENT; Model Conversion

\begin{abstract}
To integrate DIgSILENT/PowerFactory into the core computing tool of the high-level application platform for dispatching operation and planning analysis, this paper studies the automation technology of importing online grid models to DIgSILENT and exporting calculation results to DIgSILENT. Based on IEEE9 node, it studies the data content and format of grid device models, topology models and graph models in DGS interface file. A test is conducted to verify the feasibility of using DGS interface to import the entire grid models. Then it compares and analyses the online grid models in E text and the grid models in DGS interface file, studies an approach to the automatic generation of grid graphic data in DGS format based on topology data, and designs a process of converting online grid models in E text into grid models in DGS interface file. At last, this paper develops a software and tests its function. It also analyses and summaries the debugging experience of setting the node type in flow calculation. The content and achievement of this paper will play a guiding role in promoting the application of DIgSILENT for online operation analysis and offline rapid prototyping.
\end{abstract}

\section{Introduction}

With the rapid development of modern power system and the rapid building of smart grid, power system simulation has been widely applied in the field of plan, dispatch, operation and research of the grid [1]. Besides having the common analysis functions of BPA, PSASP, DIgSILENT as a large-scale integrated electric power system simulation software also has the function of visual operation and unique database management. In this way, it is easy to debug and analyze result, and provide user-defined model [2].

Although DIgSILENT has powerful and unique functions, it hasn't been applied in China's dispatch department. The main reason is the lack of DIgSILENT models data. It needs to manually build an actual large grid when using DIgSILENT as a simulation tool, which takes time and energy.

DIgSILENT provides DGS standard data interface which can be applied to exchange enormous data with other applications. Its typical applications include optional export of grid models, graphic models, or calculation results from DIgSILENT into external data source and import of complete grid models into DIgSILENT or simply update of the existing grid models.

Currently, provincial dispatch center's EMS provides online grid models in E text [3]. So this paper considers building a bridge between E text and DGS interface file, to enable automatic import of grid models into DIgSILENT.

In terms of grid models conversion, some scholars have done some research [4-10]. Research in [9] develops a data conversion program to solve the problem of onerous calculation work and being error-prone in the conversion of huge amounts of data, without generating grid graphic data, which is bad for visual operation. Research in [10] realizes BPA data's import into DIgSILENT. But it isn't a complete automatic conversion, since it needs to create grid components and graphic objects in advance. 
In order to solve the problem of lacking grid models in DIgSILENT format, this article studies a way to convert online grid models into DGS interface file which contains visual data, and provides supportive data for simulation in DIgSILENT.

\section{Research on Key Technology of Grid Models Automatically Importing into DIgSILENT Based on DGS}

\section{Grid Device Models Based on DGS}

An IEEE9 example, including electric source, two-winding transformer, load, line, capacitor and reactor, is built in DIgSILENT. Then the grid models is exported to DGS interface file. Model data in this file is as shown in Table 1.

Table 1 Device Models

\begin{tabular}{cc}
\hline Model & Description \\
\hline ElmTerm、StaCubic、StaSwitch & Bus Model, Switch Gear Model, Switch Model \\
ElmLne、TypLne & Line Model, Line Type Model \\
ElmSym、TypSym & Generator Model, Generator Type Model \\
ElmTr2、TypTr2 & Transformer Model, Transformer Type Model \\
ElmLod & Load Model \\
ElmShnt & Shunt Compensation Model \\
\hline
\end{tabular}

Grid Topology Model Data Based on DGS

Devices in DIgSILENT data model is connected by ElmTerm, StaCubic, and StaSwitch. Using a line between two buses as an example, DIgSILENT topology data model is as shown in Fig. 1.

(1) ElmTerm stands for Bus/Node;

(2) StaCubic belongs to ElmTerm and StaCubic's fold_id is consistent with the corresponding ElmTerm's ID.

(3) ElmLne's bus1 or bus2 is consistent with StaCubic's ID in the corresponding bus, and StaCubic's obj_id is consistent with ElmLne's ID, meaning that line is connected with bus.

(4) StaSwitch belongs to StaCubic and StaSwitch's fold_id is consistent with the corresponding StaCubic’s ID. Setting StaSwitch’s on_ff can change device’s operation status.

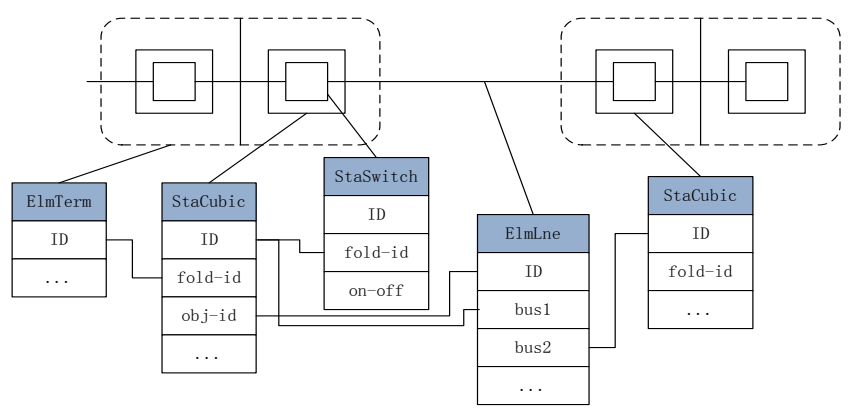

Fig. 1 Topology data models in DIgSILENT

\section{Grid Graphic Data Model Based on DGS}

Grid graphic information in DGS interface file is consisted of IntGrfcon, describing connecting line, and IntGrf, describing graphic object.

Using a line between two buses as an example, graphic data model in DIgSILENT is as shown in Fig. 2.

(1) IntGrf's sSymNam stands for the device type of graphic object and its setting is shown in Table 2.

(2) IntGrf's pDataObj is consistent with the device's ID which IntGrf represents. IntGrf's pDataObj of the line is consistent with the corresponding ElmLne's ID and IntGrf's pDataObj of bus is consistent with the corresponding ElmTerm's ID.

(3) IntGrf's rCenterX and rCenterY determine graphic object's location and are important coordinate parameters in the process of generating graphic data, which is based on grid topology. 
(4) IntGrf's other attributes are used to set color, scale and rotation of graphic object.

(5) IntGrfcon is used to connect two graphic objects. IntGrfcon between line and left bus can be a continuous broken line and IntGrfcon's $\mathrm{rX}$ and $\mathrm{rY}$ record point coordinates of broken line. IntGrfcon's fold_id is consistent with IntGrf's ID of the line.

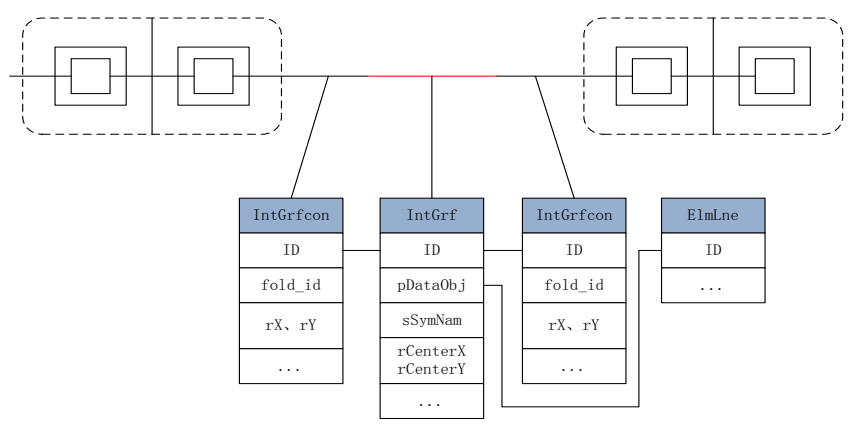

Fig. 2 Graphical data models in DIgSILENT

Table 2 Setting of sSymName

\begin{tabular}{cc}
\hline Attribute & Description \\
\hline \multirow{2}{*}{ sSymNam } & $\begin{array}{r}\text { TermStrip, Bus; d_sym, Generator; d_load, Load; d_shunt, Shunt } \\
\text { Compensator; d_lin, Line; d_tr2, Transformer }\end{array}$ \\
\hline
\end{tabular}

Test and Conclusion of Automatically Importing Whole Grid Models Based on DPL

DIgSILENT programming language DPL provides an interface for automatic programming. It import/export internal data, access or change internal object in DIgSILENT and expand the function of DIgSILENT. Based on DPL, this paper enables the automatic import of grid models and data, the transference of power flow and output of calculation results.

Using DPL, IEEE9's DGS file is automatically imported into DIgSILENT and then a new project is generated. Grid diagram and the result of power flow calculation are identical to the original grid.

Deleting graphic data in IEEE9's DGS file and importing this file into DIgSILENT generates a new project without grid diagram. But power flow calculation result is the same as the original grid.

After testing, a conclusion is made: DGS interface can enable the import of the whole grid models with/without graphic data.

\section{Key Technology of Automatically Importing Grid Models in E Text into DIgSILENT}

Comparison between Online Grid Models in E Text and Grid Models in DGS Interface File

Devices in E text have an attribute of topology node, and the topology node of device doesn't change under the same power flow interface. Therefore, network topology can be established by analyzing topology nodes.

Table 3 summaries the correspondence between online grid models in E text and grid models in DGS. But attention should be paid to the following points.

Table 3 Correspondence between E and DIgSILENT

\begin{tabular}{cc}
\hline E & DIgSILENT \\
\hline TopoNode & ElmTerm, StaCubic, StaSwitch \\
Transformer & ElmTr2, TypTr2 \\
Compensator_P & ElmShnt \\
Load & ElmLod \\
ACline & ElmLne, TypLne \\
Unit & ElmSym, TypSym \\
\hline
\end{tabular}

(1) In the process of forming bus/node model in DIgSILENT, it needs to refer to the topology connection between node and other devices. Then switchgear and switch data are added to the corresponding node to meet the DIgSILENT requirement of bus/node model. 
(2) It needs to add a new device type for every line, generator, and transformer in the conversion process.

(3) Transformer's parameters in DIgSILENT which are needed to convert with the following Eq.1 include voltage percentage of short-circuit, copper loss, no-load current, open-circuit loss.

$$
\left\{\begin{array}{l}
P_{k}=\frac{1000 S_{N}^{2} R_{T^{*}}}{U_{N}^{2}} * \frac{U_{B}^{2}}{S_{B}} \\
U_{k} \%=\frac{100 S_{N} X_{T^{*}}}{U_{N}^{2}} * \frac{U_{B}^{2}}{S_{B}} \\
P_{0}=1000 G_{T^{*}} U_{N}^{2} * \frac{S_{B}}{U_{B}^{2}} \\
I_{0} \%=\frac{100 U_{N}^{2} B_{T^{*}}}{S_{N}} * \frac{S_{B}}{U_{B}^{2}}
\end{array}\right.
$$

(4) Choosing capacity or inductance model provided by DIgSILENT depends on its operation condition in the conversion process.

\section{Method of Automatically Generating Graphic Data in DGS Format}

The grid graph can display in DIgSILENT if grid graphic data is included in DGS. The primary data of grid graphic data is location coordinates of every graphic object. But grid models data in E text has no data expressing grid graph. Based on the topology connection of devices, this article proposes a method that can automatically create location coordinators of devices and adjust locations of few devices based on the overall graphic layout.

The way of automatically creating grid graphic data in DGS is as follows.

(1) According to the node adjacency list generated by topological connection relationship of devices, the traversal sequence of nodes can be obtained by using depth-first search algorithm. The allocation of nodes' location is based on this sequence.

(2) The allocation principle of node's vertical ordinate is as follows. Nodes' vertical ordinate is same if these nodes are at the same branch. First node's vertical ordinate of forked branch increases one unit based on its parent node.

(3) The allocation principle of node's horizontal ordinate is as follows. Nodes' horizontal ordinate sequentially increases per unit if these nodes are at the same branch. First node's horizontal ordinate of forked branch increases one unit based on its parent node.

Procedure of Online Grid Models in E Text Converting into Grid Models in DGS

The three functions of model conversion module includes: grid topology reconstruction; automatic generation of grid graphic data, namely the identification of location of busbar, generator, line and transformer in DIgSILENT; models’ parameters conversion.

Based on previous study, the following conversion procedure is proposed:

(1) Deleting the data of outage and hot standby devices in order to extract the data of running devices.

(2) Forming node adjacent list based on nodes' topological connection.

(3) Choosing one node as the starting node, then using depth-first search algorithm to create a traversal sequence, which allocation of nodes' location is based on.

(4) Producing coordinators of generator, load and branch models between the nodes based on the nodes' location.

(5) Rebuilding topology in DGS interface based on original grid topology.

(6) Generating grid models’ parameters in DGS base on grid models in E text.

(7) Creating graphic data in DGS interface on the basis of procedure 4.

\section{Software Test and Debug Experience}

\section{Verification of the Function of Automatic Graph Generation}

This paper uses IEEE9 as an example to test the function of automatic graph generation. The 
network diagram produced by software is as shown in Fig. 3. Comparing with IEEE9 topology, the grid topology after conversion is the same as the original.

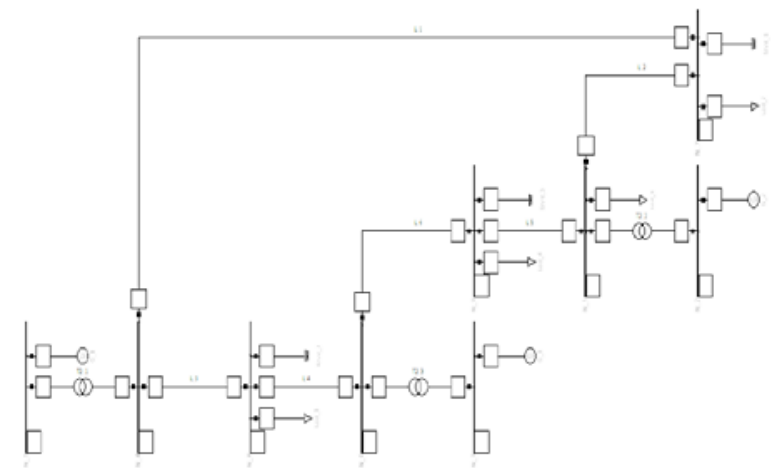

Fig. 3 Map of network topology

\section{Comparison and Analysis of Power Flow Result}

This paper verifies the accuracy of model static parameters conversion by testing the $\mathrm{E}$ text of Jibei. Grid of Jibei has 390 nodes, 366 transmission lines and 318 generators in operation. Table 4 compares the generator outputs and Table 5 shows the power flow of lines.

Table 4 Voltage and output of three units of the actual grid

\begin{tabular}{ccccccc}
\hline \multirow{2}{*}{ Generator } & \multicolumn{3}{c}{ DIgSILENT } & \multicolumn{3}{c}{$\mathrm{E}$} \\
\cline { 2 - 7 } & $\mathrm{U}$ (p.u.) & $\mathrm{P}(\mathrm{MW})$ & $\mathrm{Q}($ Mvar) & $\mathrm{U}($ p.u. $)$ & $\mathrm{P}(\mathrm{MW})$ & $\mathrm{Q}(\mathrm{Mvar})$ \\
\hline G1 & 1.02 & 577.65 & 93.45 & 1.02 & 577.64 & 72.12 \\
G2 & 1.08 & 349.92 & 91.91 & 1.07 & 340.12 & 68.29 \\
G3 & 1.00 & 210.66 & 3.18 & 1.01 & 209.54 & 3.56 \\
G4 & 1.07 & 115.22 & 129.11 & 1.07 & 115.22 & 81.12 \\
G5 & 0.99 & 17.44 & 0.02 & 1.00 & 17.00 & 0.02 \\
\hline
\end{tabular}

Table 5 Power flow of lines

\begin{tabular}{cccccc}
\hline \multirow{2}{*}{ Line } & \multirow{2}{*}{ Level $(\mathrm{kV})$} & \multicolumn{2}{c}{ DIgSILENT } & \multicolumn{2}{c}{$\mathrm{E}$} \\
\cline { 3 - 6 } & & $\mathrm{P}(\mathrm{MW})$ & $\mathrm{Q}(\mathrm{Mvar})$ & $\mathrm{P}(\mathrm{MW})$ & $\mathrm{Q}(\mathrm{Mvar})$ \\
\hline L1 & 500 & -400.96 & -77.04 & -407.48 & -61.25 \\
L2 & 500 & 338.30 & -80.07 & 340.83 & -14.10 \\
L3 & 220 & 102.69 & -23.14 & 102.70 & -22.51 \\
L4 & 220 & 61.36 & 57.02 & 60.18 & 57.03 \\
\hline
\end{tabular}

According to Table 4 and Table 5, power flow result of DIgSILENT has a little difference from real values of state estimation in E text, mainly about reactive power flow. This paper argues that the difference is caused by generator's running mode setting. Reactive power flow of the entire grid will change if the setting changes. Therefore, this paper verifies the accuracy of model static parameters conversion.

\section{Debug Experience of Power Flow Condition Setting}

Sometimes power flow that doesn't converge will occur when using the conversion data of grid models in DIgSILENT. This paper makes a conclusion that power flow convergence is mainly related with node type setting after many debugging of power flow.

In DIgSILENT, node type (PV/PQ/SL) is set by the mode of local voltage controller of the generator which connects the node. This paper identifies the following criteria to determine the mode of local voltage controller of the generator.

(1) Setting the generator node type to PV if the generator's rated power is greater than or equal to a certain value.

(2) Setting the generator node type to PQ if the generator's rated power is lower than a certain value.

(3) Setting the generator with maximum power adjustment margin in internal grid to balancing machine. 
(4) Setting the mode of equivalent generators of external network to PV operating mode.

(5) Only one generator can operate at PV mode in one single node. If more than one generators are operating at PV mode in the same node, there will be an error in power flow calculation, because a lack of coordinated control strategy will result in the unsuccessful coordinated control of the node voltage. Although DIgSILENT allows multiple generators to control the node voltage, this paper takes a simpler approach, without exploring the coordinated control strategy of multiple generators

(6) Altering PV to PQ if the power flow of PV node is irrational.

\section{Conclusion}

This article studies key technology of importing online grid models in E text into DIgSILENT, verifies the feasibility of model conversion and proposes a model conversion method. Finally, it tests IEEE9 and actual grid to verify the accuracy of the proposed method. In terms of convergence problem, debug experience is summaried in this paper.

The proposed model conversion method in this article has an exciting prospect in application because of the following points. First, using $\mathrm{E}$ text which is widely applied in dispatch department as original data solves the data collection problem. Second, it solves the problem of lacking model data in DIgSILENT and doesn't need to manually set up the whole power system. Lastly, the localization of DIgSILENT has practical significance for promoting the wide use of DIgSILENT in China's dispatch department.

\section{Acknowledgements}

In this paper, the research was sponsored by the China Electric Power Research Institute (Project No. NY71-12-010).

\section{References}

[1] China Southern Power Grid Company Limited. Simulation technology of AC and DC Power System [M]. Beijing: China Electric Power Press, 2007 3-4.

[2] Tao Lü, Zhen-xiang HAN. Introduction of power system simulation software DIgSILENT [J]. East China Electric Power, 2004, 32(12): 37-41.

[3] Yaozhong XIN, TAO Hongzhu, Yisong Li, et al. E language for electric power system model description [J]. Automation of Electric Power System, 2006, 30(10): 48-51.

[4] Longyi MA, Zhigang WU, Guanji HOU, et al. Comparison and data conversion of the steady and dynamic model for power flow and transient stability calculation in BPA and PSS/E [J]. Proceedings of the CSU-EPSA, 2010, 22(5): 128-134.

[5] Hua TAO, Jinjin XU, Wencong ZHOU. Model conversion from BPA to PSCAD [J]. Electric Power Automation Equipment, 2013, 33(8): 152-156.

[6] Min ZHANG, Renmu HE, Jinjin XUN, et al. Application of Graph Theory in Approach for Automatically Converting BPA Models into PSCAD/EMTDC Models [J]. Power System Technology, 2012, 36(6): 163-169.

[7] ORREGO N J, BEDOYA V H. Loading procedure to transfer an Electrical Network Data Base from DIgSILENT PowerFactory to CAPE [C] //ANDESCON, 2010 IEEE. IEEE, 2010: 1-5.

[8] UBISSE A V, FOLLY K A, AWODELE K O, et al. Comparison of Matlab PST, PSAT and DIgSILENT for transient stability studies on parallel HVACHVDC transmission lines [C]// Universities Power Engineering Conference (UPEC), 2010 45th International.

[9] Tao Lü. Use and compare of power system simulation software [D]. Hangzhou: Zhejiang University, 2005.

[10] Qing LIU, Dongying ZHANG, Yanhua LIU, et al. Research and development of BPA grid model imported to DIgSILENT [J]. 2014, 16: 112-117. 